\title{
A Perceptual Heuristic for Shadow Computation in Photo-Realistic Images
}

\author{
Peter Vangorp Olivier Dumont Toon Lenaerts Philip Dutré \\ Department of Computer Science, Katholieke Universiteit Leuven*
}

Perceptual research in photo-realistic rendering has mainly focused on the limitations of the human visual system to predict visible differences between the pixels of a radiometrically correct image and an approximation of the same image [Daly 1993]. However, research in perceptual psychology and vision suggests that image interpretation is inherently a higher-level, feature-based process. Furthermore, applications rarely demand a direct comparison to a radiometrically correct image, so approximations can be less strict. We propose a novel approach to perception that is geared towards these conditions for observing images.

\section{Perceptual experiment}

This sketch presents a perceptual heuristic for the approximation of cast shadows in photo-realistic image synthesis. Previous work has proven that the presence of shadows provides important visual clues for the interpretation of a scene [Kersten et al. 1994]. We conducted an initial perceptual experiment to measure the importance of realistic shadows. Subjects were asked to judge the perceived realism for different configurations of the light source, occluder and shadow receiver in a simple Cornell box scene. In some stimuli the shadow was rendered correctly, in others it was completely omitted (Figure 1(a)).
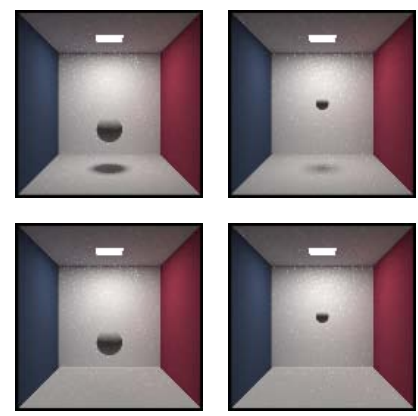

(a)

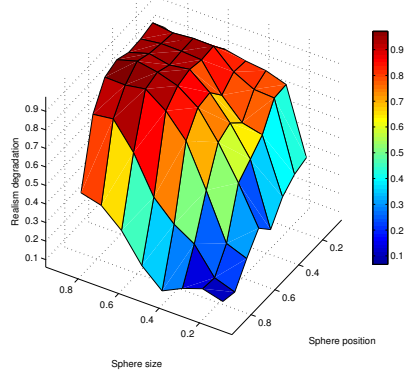

(b)

Figure 1: Initial experiment. (a) Some of the stimuli used. (b) Derived heuristic as a function of occluder size and position.

Based on this experiment, a heuristic was developed that expresses the probability with which approximations will be perceived to be less realistic than correct shadows (Figure 1(b)). This figure shows that the realism of the approximate image decreases more for larger occluders or occluders closer to the shadow receiver. When translated into visual effects, the heuristic supports the observation that an accurate rendering of the presence of an umbra will be much more important than that of a penumbra.

\section{Perceptually realistic rendering}

The heuristic was built into a global illumination algorithm to decide when shadows can be approximated, without affecting the re-

\footnotetext{
*e-mail: \{peter.vangorp,olivierd,toon.lenaerts,phil\}@cs.kuleuven.be
}

alism of the image. We chose to implement a continuous approximation scheme that produces noiseless and perceptually convincing shadows. With this modification the generalized heuristic can be validated in another experiment similar to the original one.

The heuristic provides spatially consistent decisions for featurebased approximations in an otherwise pixel-based rendering algorithm. A modified shadow photon map [Jensen 2001] was used to store the precomputed heuristic values in the scene. Each shadow photon stores the heuristic value calculated for its light source and occluder. A user-specified threshold defines which shadows are approximated. In our experience, up to $50 \%$ of the pixels in an image may be approximated without noticeable loss of realism, depending on the scene and the threshold parameter. Of course, when comparing to a correct image, differences probably would be noticeable. Note that this is not a limitation of the algorithm, but is exactly what our approach is designed to do.

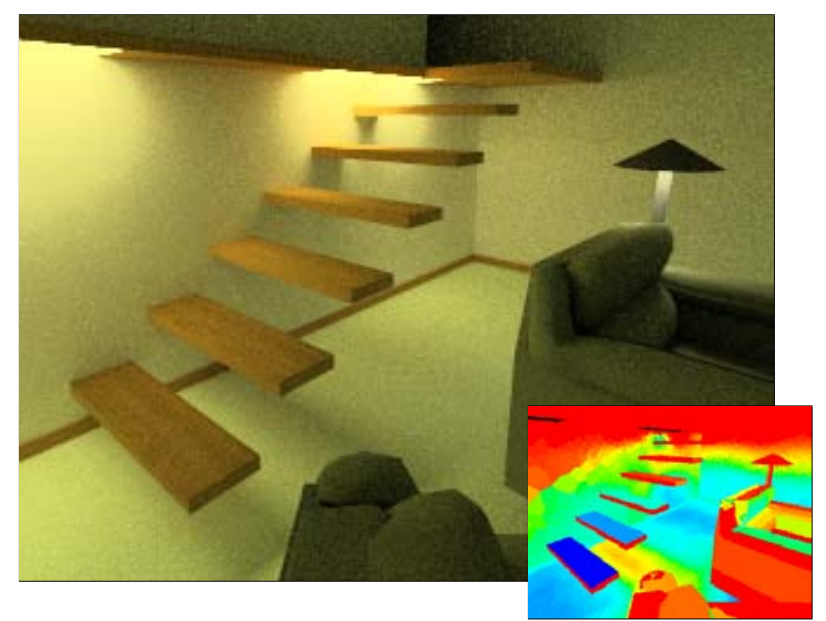

Figure 2: A scene rendered with approximate shadows. Inset: false color image of the heuristic. The shadows in the blue-green region under the staircase can be approximated.

As future work, we would like to extend this approach to other illumination effects in a scene, such as highlights or caustics. Similar perceptual tests could provide insights into the impact on realism of shader simplification or modified rendering algorithms.

\section{References}

DALY, S. 1993. The visible differences predictor: an algorithm for the assessment of image fidelity. Digital images and human vision. MIT Press, 179-206.

Jensen, H. W. 2001. Realistic Image Synthesis Using Photon Mapping. A. K. Peters, Natick, MA, USA.

Kersten, D., Mamassian, P., And Knill, D. 1994. Moving cast shadows and the perception of relative depth. Tech. Rep. CogSci Memo 6, Max Planck Institut für biologische Kybernetik. 